\title{
Anabases
}

ANABASES Traditions et réceptions de l'Antiquité

$17 \mid 2013$

Varia

\section{David KARMON, The Ruin of the Eternal City. Antiquity and Preservation in Renaissance Rome}

\section{Anne-Hélène Klinger-Dollé}

\section{(2) OpenEdition}

\section{Journals}

Édition électronique

URL : http://journals.openedition.org/anabases/4268

DOI : $10.4000 /$ anabases.4268

ISSN : 2256-9421

\section{Éditeur}

E.R.A.S.M.E.

\section{Édition imprimée}

Date de publication : 1 mars 2013

Pagination : 283-286

ISSN : 1774-4296

\section{Référence électronique}

Anne-Hélène Klinger-Dollé, "David kaRmon, The Ruin of the Eternal City. Antiquity and Preservation in Renaissance Rome », Anabases [En ligne], 17 | 2013, mis en ligne le 01 avril 2013, consulté le 22 septembre 2020. URL : http://journals.openedition.org/anabases/4268 ; DOI : https://doi.org/ $10.4000 /$ anabases. 4268

Ce document a été généré automatiquement le 22 septembre 2020

(c) Anabases 


\title{
David KARMON, The Ruin of the Eternal City. Antiquity and Preservation in Renaissance Rome
}

\author{
Anne-Hélène Klinger-Dollé
}

\section{RÉFÉRENCE}

David KARMON, The Ruin of the Eternal City. Antiquity and Preservation in Renaissance Rome, Oxford, Oxford University Press, 2011, 320 p., 40 livres / isbn 978-0-19-976689-5.

1 Il n'est guère difficile de rendre compte de la démarche et de la structure de cet ouvrage, malgré sa richesse, tant le propos de David Karmon (D.K.) est clair et structuré. Dans l'introduction, sa thèse est exposée très nettement. L'historiographie qui a prévalu dès les écrits humanistes, et qui a pris une forme définitive dans la Storia degli scavi di Roma de Rodolfo Lanciani (1902-1912), dépeint la Rome papale de la Renaissance comme s'étant livrée à un véritable pillage de la ville antique. Alors même que l'Antiquité était l'objet d'études et de découvertes ferventes, la fièvre bâtisseuse de la Renaissance se rendait coupable des pires destructions. Comme le dit l'auteur, cette vision fait encore autorité dans l'ouvrage de référence que constitue The Renaissance Discovery of Classical Antiquity de Roberto Weiss (1969), où on la voit apparaître incidemment. Or c'est justement cette lecture historiographique qu'entend discuter The Ruin of the Eternal City. La démonstration s'attache donc à rouvrir la documentation existante, en la complétant parfois de nouveaux documents d'archives, et à traquer tous les indices de ce que l'auteur appelle des preservation practices, dont la Rome antique et médiévale avait déjà donné des exemples et dont la Renaissance, selon lui, a eu le souci à un degré plus poussé que jamais. L'auteur explique d'emblée qu'il préfère le terme de preservation à celui de conservation. Ce dernier, selon lui, est trop étroitement associé à nos représentations modernes de ce que doit être la conservation $\mathrm{du}$ patrimoine pour nous permettre de comprendre la logique propre de pratiques qui 
ne reposent pas sur les mêmes présupposés théoriques. Il s'agit bien moins de vouloir stabiliser l'état d'un bâtiment et préserver le passé que de le réanimer, de le revivifier en fonction des attentes du présent, y compris par des interventions importantes. Les sources sur lesquelles s'appuie D.K. sont de trois ordres : l'étude archéologique des sites et bâtiments eux-mêmes, les nombreuses représentations (dessins, gravures) élaborées à la Renaissance, reproduites très largement dans l'ouvrage, enfin des documents d'archives, en particulier du Vatican et du Capitole.

2 L'ouvrage s'articule en deux parties. La première s'intitule A History of Preservation Practices in Renaissance Rome. Le premier chapitre pointe de manière synthétique quelques mesures de "préservation » antiques et médiévales, autour de deux périodes importantes pour l'histoire de la Ville et son rapport avec le patrimoine: Auguste, d'une part, la formation d'un gouvernement municipal en 1144 d'autre part. L'auteur s'attache à mettre en évidence les implications politiques des mesures prises. Qu'il s'agisse du princeps Auguste ou du gouvernement municipal soucieux d'apparaître comme l'héritier du sénat antique, l'enjeu est toujours d'asseoir sa légitimité politique en se faisant le protecteur de la Ville et de son patrimoine. Le deuxième chapitre commence avec le retour de Martin V à Rome en 1420, vécu comme une véritable instauratio, après la désertion des papes pour Avignon. En 1425, une bulle de Martin V met les maestri di strade et degli edifici, qui avaient été créés au XII siècle par le gouvernement municipal, sous l'autorité directe du pape. L'évocation des règnes d'Eugène IV, de Nicolas V (le " pape des humanistes »), de Pie II, montre comment deux pouvoirs en rivalité, la papauté et les conservateurs municipaux, cherchent chacun à s'affirmer sur le terrain de la preservation, notamment dans l'octroi de licences d'exploitation de pierres sur les sites antiques. Cette législation est souvent balayée d'un revers de main par l'historiographie traditionnelle, qui dénonce sa totale impuissance à préserver réellement les sites en question. D.K. invite à une lecture plus nuancée, montrant l'évolution de cette législation dans un sens de plus en plus précis et restrictif. Il lui semble aussi possible de faire apparaître une évolution progressive des mentalités. Depuis l'Antiquité, les ruines étaient considérées comme un signe de décadence et la législation du début du Quattrocento fait une différence nette entre les ruines, qu'on peut exploiter sans vergogne, et les parties intactes d'un monument, qui doivent être préservées. Au contraire, la Bulle de 1462 promulguée par Pie II manifeste un intérêt qui s'étend aux parties ruinées des édifices, ce qui ouvre la voie à des pratiques plus larges de préservation. Le chapitre 3 procède lui aussi à l'étude fouillée des documents d'archives, particulièrement des licences concédées, pour la période qui va de l'élection de Jules II en 1503 à la mort de Paul III en 1549. Sur les dix-sept documents, italiens et surtout latins, reproduits en fin de volume et traduits en anglais, seize concernent cette période et sont de lecture passionnante. Le chapitre accorde une attention toute particulière à la célèbre lettre de Raphaël et Castiglione à Léon $\mathrm{X}$, véritable plaidoyer en faveur de la préservation de l'Antiquité. L'auteur rappelle alors les tensions, plutôt que les contradictions, qui traversent la Renaissance - mais aussi toute époque selon lui - entre désir de préserver et nécessité (ou passion) de bâtir une ville moderne, qui célèbre une puissance retrouvée. Le chapitre s'achève enfin sur les immenses travaux de dégagement des sites antiques réalisés par Paul III pour la venue de Charles Quint en 1536. Ces travaux ont façonné en grande partie les vues modernes que nous avons du Forum et des grands monuments antiques : elles ont mis en valeur les vestiges, tout en bouleversant la cité. 
3 La deuxième partie du livre, intitulée Object biographies, est une série de trois études consacrées à l'histoire de la préservation, qui tantôt a réussi, tantôt s'est soldée par un échec, de trois monuments romains de nature très différentes : le Colisée, le Panthéon, le pont Santa Maria. D.K. ouvre volontairement ces études monographiques par le Colisée, témoin par excellence des dégradations du temps, en sa partie sud, et de l'exploitation de ses matériaux. L'auteur s'attache à montrer que même dans ce cas-ci, des mesures limitatives ont existé et ont été respectées. Le Panthéon représente a priori le cas inverse par son état de conservation remarquable. L'auteur montre que cette préservation, loin de relever du hasard, est le fruit de l'implication soutenue des papes, notamment en ce qui concerne la toiture. Enfin, le dernier chapitre s'intéresse à la préservation d'un monument qui s'est effondré en 1557, et dont il ne reste aujourd'hui qu'une ruine : le pont Santa Maria. Il s'agit du premier pont de pierre de Rome, édifié au $\mathrm{II}^{\mathrm{e}}$ s. av. J.-C. C'est un lieu important d'un point de vue économique, mais aussi religieux. D.K. montre comment il a fait l'objet des soins conjoints des papes et des grandes familles romaines au long des siècles. Les travaux de restauration furent particulièrement nombreux et important dans les années 1540-1550 et le chantier fut un temps sous la direction de Michel Ange. D.K. avance la thèse que l'échec de ces restaurations n'est pas tant à mettre au compte du successeur et rival de Michel Ange, Nanni, qu'aux closes très restrictives qui lui étaient imposées, demandant de respecter l'architecture originelle du pont - peut-être pour des raisons avant tout financières. Alors que cette restauration semble plus proche de nos normes de conservation modernes, le résultat ne fut pas probant.

4 La conclusion de l'ouvrage suggère l'intérêt des questions soulevées par cette manière différente de lire l'histoire des vestiges antiques. Il s'agit de réfléchir davantage aux rapports dialectiques existant entre destruction et préservation, en montrant que l'opposition n'est jamais nette et tranchée. L'auteur propose aussi que le concept de " créativité » en architecture ne se concentre pas uniquement sur la construction de nouveaux bâtiments, mais aussi sur les processus qui permettent la vie d'un édifice au cours des siècles. D.K. revient sur le titre de son livre et propose de voir ce qui fait l'« éternité » de Rome dans l'équilibre qu'elle n'a cessé de recréer à différents moments de son histoire entre la conservation scrupuleuse du passé et les changements nécessaires à la vie.

5 Ce livre très documenté se lit avec aisance et grand intérêt. Ses points de vue sont toujours stimulants en même temps qu'exposés avec clarté. Parfois, l'argumentation de tel ou tel point de détail n'a pas emporté réellement notre conviction. Ainsi, il nous semble difficile de pouvoir en toute rigueur assimiler le Colisée à une relique parce que le sacristain de Saint-Pierre a demandé des pierres du Colisée pour réaliser un ouvrage destiné à abriter la Pietá de Michel Ange. Le propos est peut-être parfois un petit peu trop martelé à notre goût. La Rome de la Renaissance est par ailleurs très majoritairement vue sous l'angle de la Rome papale. Certes, le rôle d'autres personnages, notamment des cardinaux et de grandes familles, apparaît ponctuellement à l'occasion de l'examen des licences d'extraction. Mais il nous semble que ces autres acteurs possibles des transformations de la ville renaissante restent assez largement dans l'ombre. Il n'en demeure pas moins que toute cette enquête est passionnante, érudite et très riche pour mieux comprendre les comportements propres à la Renaissance à l'égard du patrimoine antique et leur raison d'être. 


\section{AUTEURS}

ANNE-HÉLÈNE KLINGER-DOLLÉ

Université de Toulouse (UTM)

dolle@univ-tlse2.fr 